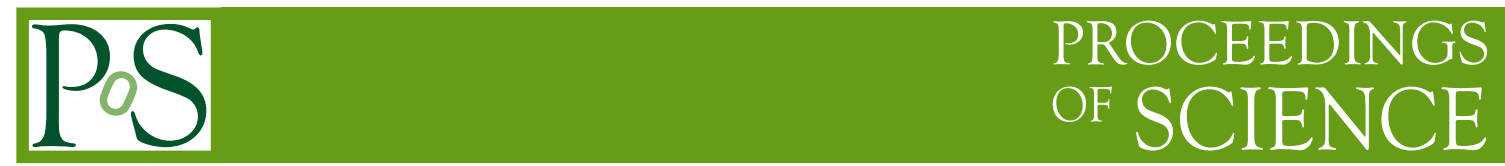

\title{
Searches for Dark Matter at the LHC
}

\author{
John Butler* \\ Boston University \\ E-mail: jmbutlerabu.edu \\ on behalf of the ATLAS, CMS and LHCb Collaborations
}

\begin{abstract}
The existance of a new form of matter, Dark Matter, has been established by a large body of astrophysical measurements. The particle nature of Dark Matter is one of the most intriguing and important open issues in physics today. A review of searches for Dark Matter by the ATLAS, CMS and LHCb experiments is presented.
\end{abstract}




\section{Introduction}

Astrophysical observations provide compelling evidence for a new type of matter, Dark Matter (DM), via its gravitational interactions. Measurements reveal that DM is five times more abundant than ordinary baryonic matter [1]. The evidence indicates that DM is not a Standard Model (SM) particle and is therefore de facto new physics. One of the most important open questions in physics today is uncovering the particle nature of DM. A leading candidate for DM is a Weakly Interacting Massive Particle (WIMP). A WIMP is an electrically neutral, colorless, stable particle with a mass in the range of the electroweak scale, of order $100 \mathrm{GeV}$. The "WIMP Paradigm" is highly attractive as it naturally accounts for the DM relic density in the universe observed today. Most of the results presented here will assume WIMP DM.

The DM-SM interaction can be probed in several complementary and interdisciplinary ways. Direct Detection (DD) experiments look for evidence of DM-nucleus elastic scattering. Indirect Detection (ID) experiments search for SM particles from DM annihilation or decay. The ATLAS [2], CMS [3] and LHCb [4] experiments at the Large Hadron Collider (LHC) rely on detecting dark-sector particles produced in $p p$ collisions. Results from the LHC are covered here, more on DD and ID experiments was shown in several other presentations at this meeting. Unless otherwise noted, the LHC results that follow are based on the 2015/16 LHC Run 2 dataset at $\sqrt{s}=13 \mathrm{TeV}$.

The DM "theory space" is populated by a large number of models that can be classified in terms of how complete they are theoretically, see for example [5] for a review. At the "less complete" end of the spectrum are models based on Effective Field Theories (EFTs). The EFT describes a universal DM-SM interaction mediated by kinematically inaccessible particles, basically a contact interaction, and the DM is the only dark-sector particle probed by experiment. The "more complete" end of the spectrum includes models based on SUSY, Universal Extra Dimensions and Little Higgs which are more theoretically sound but the realization of DM is highly model dependent.

In between EFT models and more complete models reside "Simplified" DM models. All the LHC DM searches described in the following are based on models of this type. Simplified models are characterized by two states: DM and a mediator. These models resolve the EFT contact interaction into an $s$ - or $t$-channel exchange of the mediator. Compared to a EFT models, simplified models have more parameters, including the couplings of the mediator to SM and DM particles, the DM mass and the mediator mass. The LHC experiments generally quote results for baseline couplings scenarios that were proposed by the LHC DM Working Group [6]. The DM particle $\chi$ is typically taken to be a Dirac fermion. The mediator can be spin-1, with vector or axial-vector couplings, or spin- 0 , with scalar or pseudoscalar couplings. The coupling of the spin-1 mediators to quarks is universal and the spin-0 mediators have Yukawa couplings like the SM Higgs. These interactions guarantee the models obey Minimal Flavor Violation (MFV) to evade constraints from flavor physics.

\section{Searches for DM with $\left(E_{\mathbf{T}}^{\text {miss }}+X\right)$ Signatures}

When DM is produced at colliders, it escapes without detection which results in an imbalance in transverse momentum $\vec{p}_{\mathrm{T}}$. The magnitude of the missing $\vec{p}_{\mathrm{T}}$ is denoted $E_{\mathrm{T}}^{\text {miss }}$. Many DM searches 
at the LHC exploit this " $E_{\mathrm{T}}^{\text {miss }}+X$ " or "Mono- $X$ " signature where $X$ stands for SM particles that tag the event. Searches in this channel include $X$ being a jet, a photon, a vector boson ( $W$ or $Z$ ), heavy flavor quark ( $t$ or $b$ quark) and a Higgs boson $h$. The results presented next assume a single mediator and the DM is a Dirac fermion $\chi$. The parameters of the model are the mass of the mediator $m_{\text {med }}$, the DM mass $m_{\chi}$, the coupling of the mediator to quarks and DM, $g_{q}$ and $g_{\chi}$ respectively.

\subsection{Searches in the $\left(E_{\mathbf{T}}^{\text {miss }}+\right.$ jet $)$ Channel}

The most sensitive Mono- $X$ channel is $\left(E_{\mathrm{T}}^{\text {miss }}+\right.$ jet $)$ where the signature is a high $p_{\mathrm{T}}$ jet from initial state radiation (ISR) and large $E_{\mathrm{T}}^{\text {miss }}$ from DM. The final discriminant is the distribution of $E_{\mathrm{T}}^{\text {miss }}$ where DM would produce an excess over SM expectations, particularly at high $E_{\mathrm{T}}^{\text {miss }}$. The main backgrounds are $Z(v v)+$ jets and $W(l v)+$ jets which are constrained by control regions. Results of $\left(E_{\mathrm{T}}^{\text {miss }}+\right.$ jet $)$ searches from ATLAS and CMS are shown in Figure 1 assuming an axialvector mediator (left) or a vector mediator (right). For couplings $g_{q}=0.25$ and $g_{\chi}=1.0$, axialvector and vector mediators excluded up to $1.8 \mathrm{TeV}(1.55 \mathrm{TeV})$ by CMS [7] (ATLAS [8]) for $m_{\chi} \sim 1 \mathrm{GeV}$.
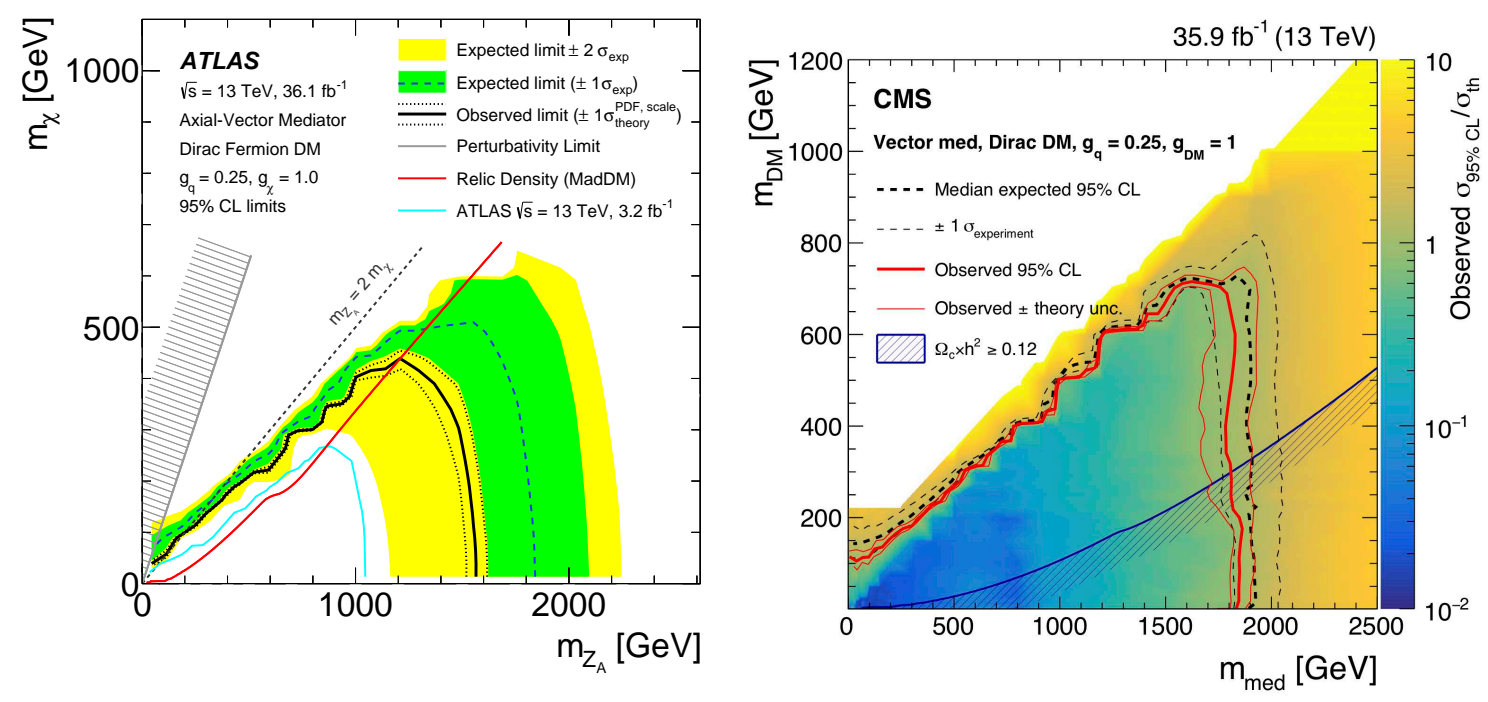

Figure 1: Exclusion regions in the DM mass-mediator mass plane from searches in the $\left(E_{\mathrm{T}}^{\mathrm{miss}}+\right.$ jet $)$ channel: (left) from ATLAS for an axial-vector mediator [8] and (right) from CMS for a vector mediator [7].

The results displayed in Figure 1 can be reinterpreted in terms of the DM-nucleon crosssection as a function of $m_{\mathrm{DM}}$ to allow comparison with DD experiments. Figure 2 shows limits on DM-nucleon cross-section assuming a spin independent interaction $\left(\sigma_{\mathrm{DM}-\text { nucleon }}^{\mathrm{SI}}\right)($ left $)$ and a spin dependent DM-proton interaction $\left(\sigma_{\mathrm{SD}}(\chi\right.$-proton $\left.)\right)$ (right). The LHC results are complementary to those from obtained from DD, in particular with superior sensitivity to DM masses below the detection threshold in DD experiments. Comparing LHC and DD limits must be done with care, the LHC limits are only valid for the specific model parameters shown.

Limits on $\sigma_{\mathrm{DM}-\text { nucleon }}^{\mathrm{SI}}$ are improved by CMS [9] by interpreting the limit on the branching fraction of the Higgs boson decaying to invisible particles, $B(\mathrm{H} \rightarrow \mathrm{inv})<0.2$ at $90 \% \mathrm{CL}$ in the 

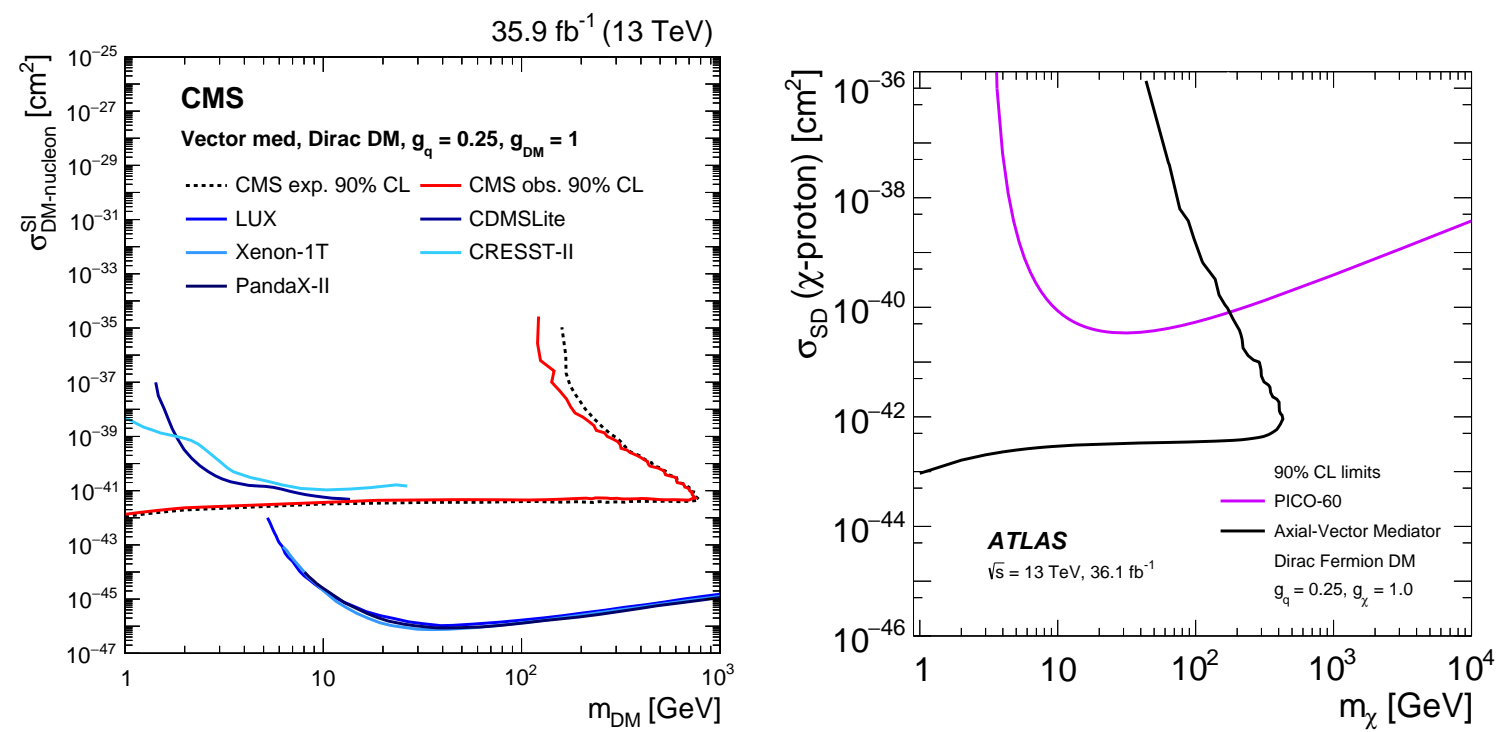

Figure 2: Comparison of LHC results from searches in the $\left(E_{\mathrm{T}}^{\mathrm{miss}}+\right.$ jet $)$ channel with results from DD experiments: (left) the limit on the spin-independent DM-nucleon cross-section vs. DM mass from CMS [7] and (right) the limit on the spin-dependent $\chi$-proton cross-section vs. DM mass from ATLAS [8].

context of a Higgs-portal DM model. The results shown in Figure 3 (left) provide limits on a fermion (scalar) $\chi$ for $m_{\chi}<20(7) \mathrm{GeV}$.

In contrast to the $\left(E_{\mathrm{T}}^{\mathrm{miss}}+\right.$ jet $)$ searches just discussed where a colorless spin 1 mediator is exchanged in the $s$-channel, it is possible for a mediator with a $q \chi$ coupling to be exchanged in the $t$-channel. ATLAS has searched for a scalar colored mediator $\eta$ in the $\left(E_{\mathrm{T}}^{\mathrm{miss}}+\right.$ jet $)$ final state where the jet comes from either ISR or from the $\eta$ decay. In the model considered, the $\eta$ couples to the first two generations of quarks and the model parameters are $m_{\eta}, m_{\chi}$ and $g \equiv g_{q \chi}=1$. The results of a search by ATLAS [8] are shown in Figure 3 (right) where mediator masses are excluded up to $1.67 \mathrm{TeV}$ for low $m_{\chi}$. For $m_{\chi}=m_{\eta}$, masses up to $620 \mathrm{GeV}$ are excluded.

\section{Searches for Mediators}

It has been said that the "LHC is a mediator machine" in that mediators rather than DM are directly produced by the interaction. Since the mediator couples to SM particles, it can also decay to SM particles and this motivates searches for the mediator in dijet and dilepton final states. The signature for the dijet search is two high $p_{\mathrm{T}}$ jets and the discriminant is the dijet invariant mass $m_{j j}$. The analysis looks for a bump on the smoothly falling $m_{j j}$ distribution which is modeled by a parameterized function. Results of searches from ATLAS [10] and CMS [11] are shown in Figure 4 assuming an axial-vector mediator (left) or a vector mediator (right). The excluded region depends strongly on the couplings, limits in Figure 4 assume the benchmark couplings $g_{\mathrm{DM}}=1, g_{q}=0.25$ and $g_{l}=0$. No leptonic coupling means the analysis is the same as for leptophobic $Z^{\prime}$ searches. Dijet searches significantly extend the DM reach, particularly for $m_{\mathrm{DM}}>M_{\text {med }} / 2$. Mediator masses are excluded up to about $2.6 \mathrm{TeV}$ for low $m_{\chi}$. 

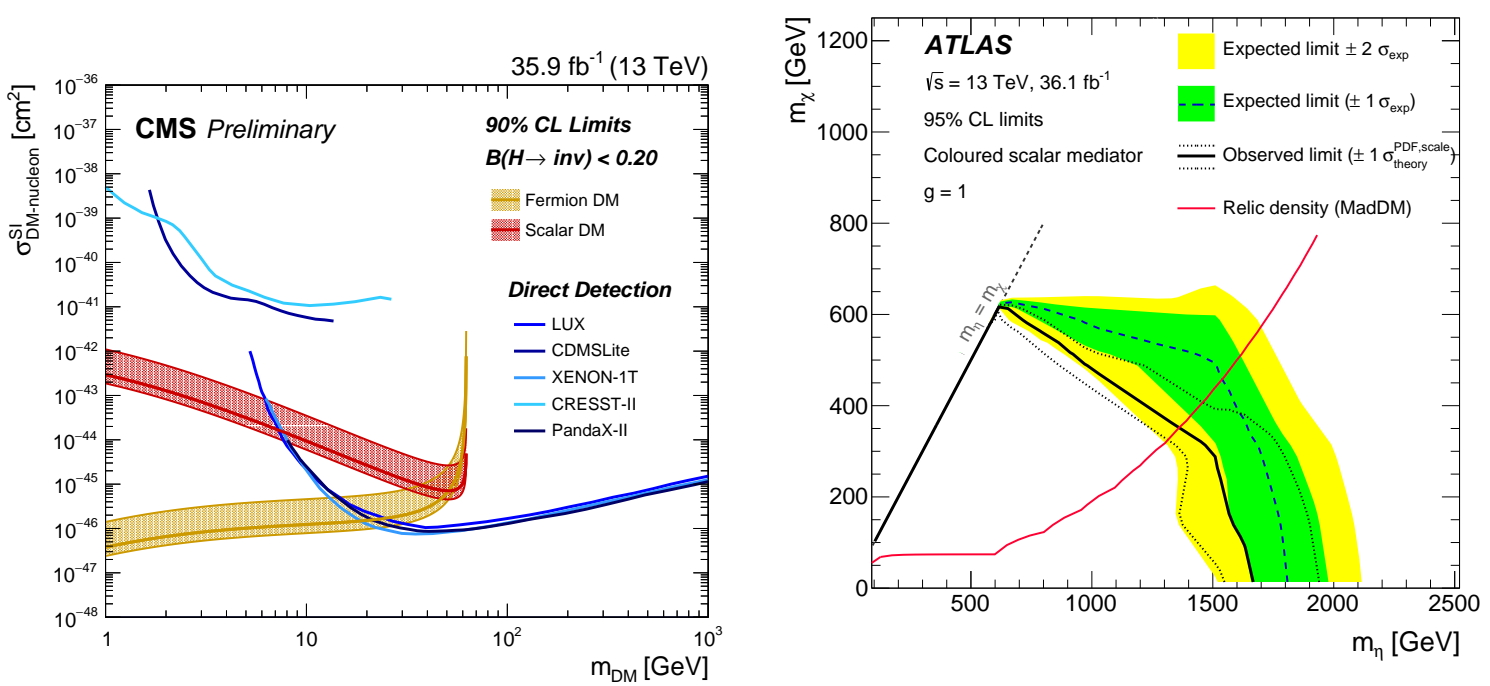

Figure 3: (Left) the limit on the spin-independent DM-nucleon cross-section vs. DM mass from CMS [9], (Right) exclusion region in the DM mass-mediator mass plane from the ATLAS search [8] for a scalar colored mediator $\eta$.
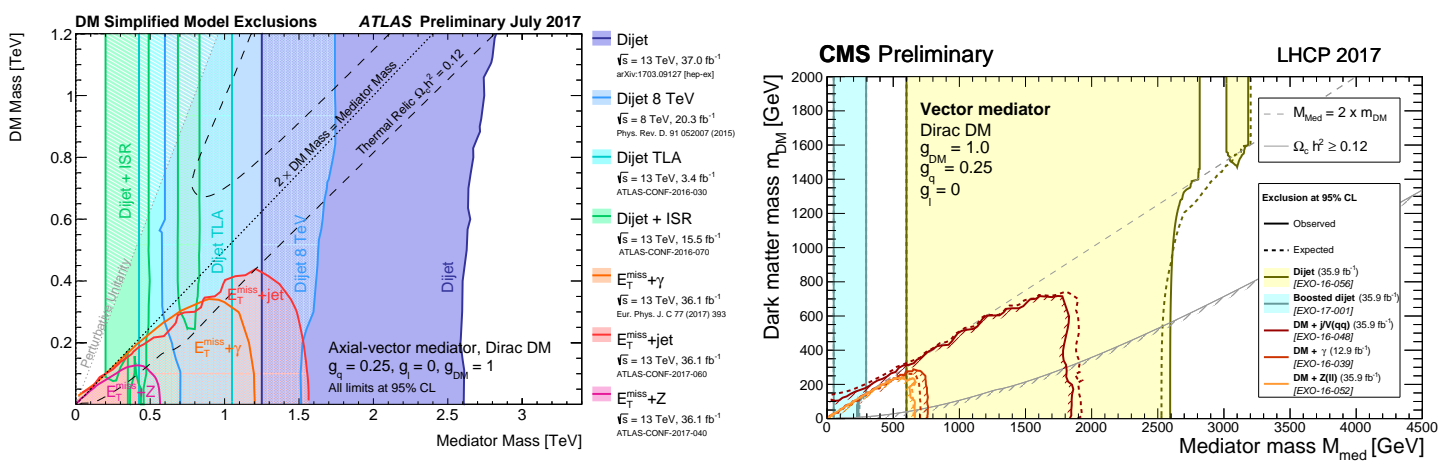

Figure 4: Compilation of exclusion regions in the DM mass-mediator mass plane: (left) from ATLAS for an axial-vector mediator [10] and (right) from CMS for a vector mediator [11].

Dijet searches excel at probing high mediator masses but the sensitivity at low $m_{j j}$ is limited by the jet trigger. Unprescaled single jet triggers have high $p_{\mathrm{T}}$ thresholds while low $p_{\mathrm{T}}$ threshold triggers suffer from significant prescaling. New techniques have been developed to trigger on low $p_{\mathrm{T}}$ dijet events. One approach is known as "data-scouting" in CMS and "Trigger-object Level Analysis (TLA)" in ATLAS. The basic idea is to use a reduced data format to allow a high trigger rate while maintaining a modest bandwidth. Sensitivity to even lower mediator masses is achieved by requiring hard ISR (jet or photon) in addition to a light dijet resonance. Figure 5 shows limits from CMS [12] (left) and ATLAS [13] (right) that exploit these techniques.

\section{Search for Dark Matter Plus Heavy Flavor}

To be consistent with MFV, spin-0 mediators (the scalar $\phi$ and the pseudoscalar $a$ ) have SM 

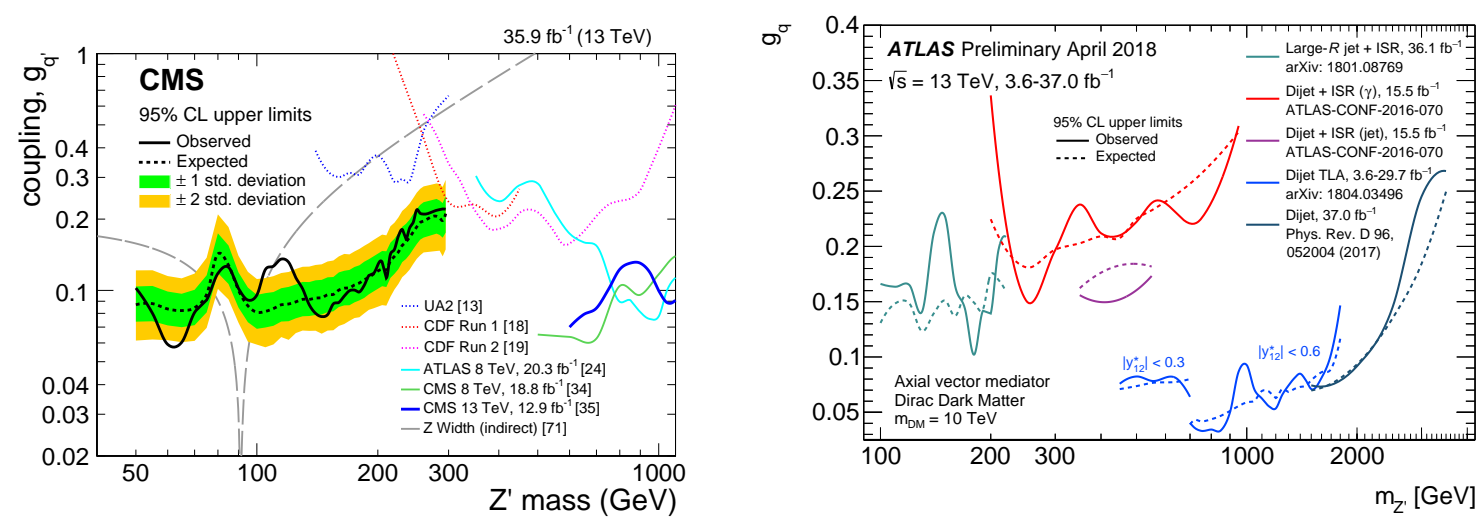

Figure 5: Compilation of limits on the mediator coupling to quarks $g_{q}$ vs. mediator mass: (left) from CMS [12] and (right) from ATLAS [13]

Higgs-like Yukawa couplings which means that they couple most strongly to $t$ and $b$ quarks. This motivates looking for $\phi / a \rightarrow \chi \bar{\chi}$ in final states with heavy flavor quarks and large $E_{\mathrm{T}}^{\text {miss }}$. CMS has searched for $p p \rightarrow t \bar{t}+\chi \bar{\chi}$ [14] using multiple signal regions based on the $t \bar{t}$ decays (allhadronic, $\ell+$ jets and dileptonic) which provide complementary sensitivity. The $t \bar{t}, W+$ jets and $Z+$ jets backgrounds are constrained by control regions. Results are shown in Figure 6 where, for couplings $g_{q}=1, g_{\mathrm{DM}}=1$ and $m_{\chi}=1 \mathrm{GeV}$, CMS excludes $m_{\phi}<165 \mathrm{GeV}$ and $m_{a}<223 \mathrm{GeV}$.
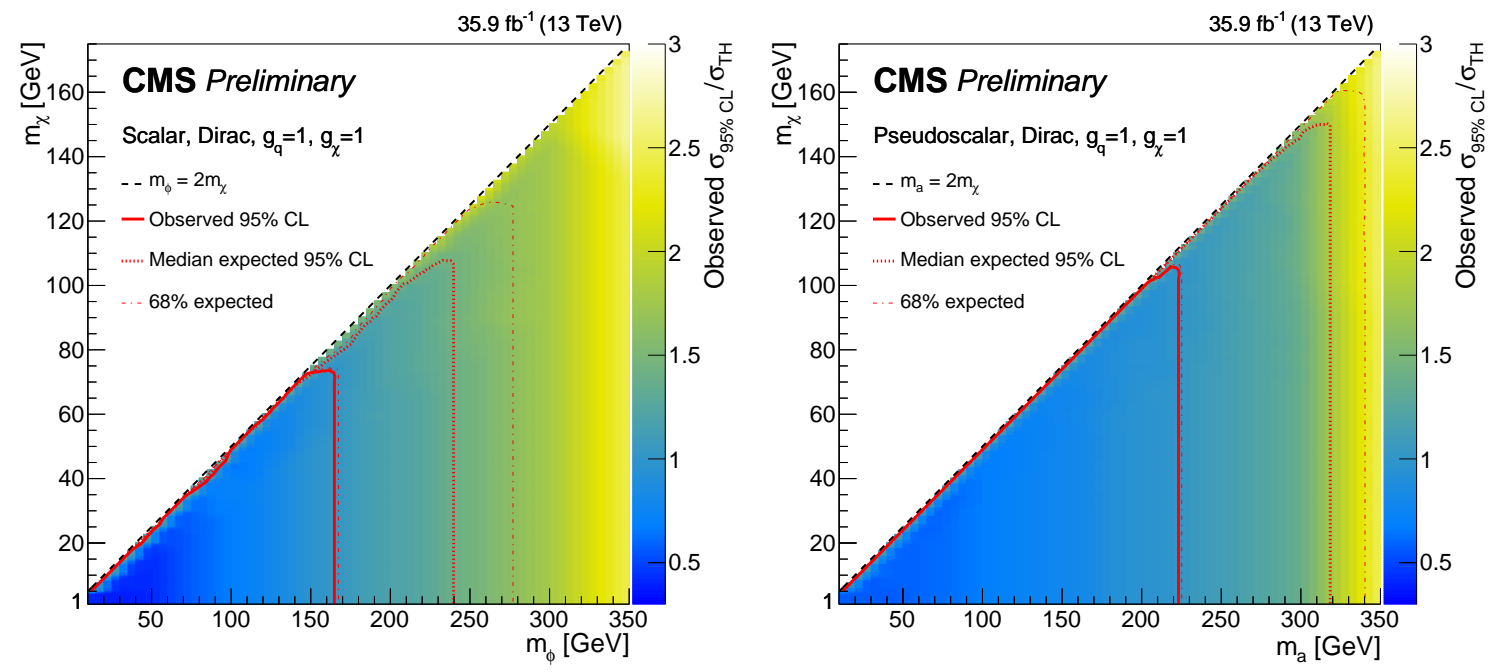

Figure 6: Exclusion regions in the DM mass-mediator mass plane from the CMS search in the $t \bar{t}+\chi \bar{\chi}$ channel [14]: (left) for a scalar mediator and (right) for a pseudoscalar mediator.

\section{Searches in the $\left(E_{\mathbf{T}}^{\text {miss }}+\right.$ Higgs $)$ Channel}

The discovery of the Higgs boson $h$ enables new avenues for DM searches in the $\left(E_{\mathrm{T}}^{\text {miss }}+h\right)$ final state. For a SM-like Higgs boson, the couplings of $h$ to initial state quarks are Yukawa suppressed and so $h$ does not come from ISR but from its coupling to the mediator. A number of 
models in the literature produce the $\left(E_{\mathrm{T}}^{\text {miss }}+h\right)$ signature, recent results from two such models are presented here. In the "Baryonic $Z$ '" model, the mediator $Z$ ' is produced in the $s$-channel, it radiates the $h$ and then decays $Z^{\prime} \rightarrow \chi \bar{\chi}$. CMS has searched for this process where the $h$ decays to either $\gamma \gamma$ or $\tau^{+} \tau^{-}$[15]. Results are shown in Figure 7 (left) where the $Z^{\prime}$ is excluded up to $815 \mathrm{GeV}$ for low $m_{\chi}$. ATLAS has searched for DM in the context of a $Z^{\prime}$-2HDM model where $Z^{\prime} \rightarrow h A$ followed by the pseudoscalar Higgs $A$ decaying to $\chi \bar{\chi}$. The Higgs boson decays to $b \bar{b}$ and is reconstructed using resolved or merged jets [16]. Results are presented in the $m_{A}-m_{Z^{\prime}}$ plane in Figure 7 (right) where $Z^{\prime}$ is excluded up to $2.6 \mathrm{TeV}$ and $A$ up to $600 \mathrm{GeV}$ for the chosen model parameters. The $Z^{\prime}$-2HDM result from CMS was presented by A. Spiezia at this meeting.
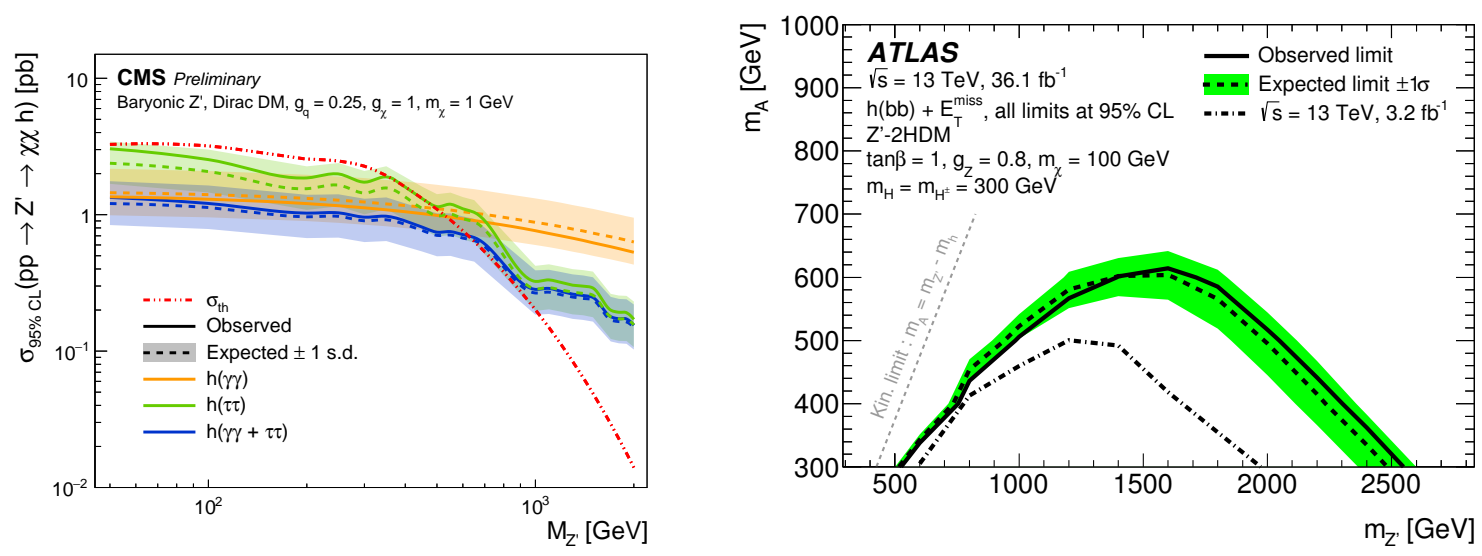

Figure 7: (Left) Limit on cross-section vs. mediator mass for the Baryonic $Z^{\prime}$ models from CMS [15]. (Right) Exclusion region in the $m_{A}-m_{z^{\prime}}$ plane in the context of the $Z^{\prime}$-2HDM model from ATLAS [16].

\section{Search for Dark Photons}

There have been numerous searches for DM as WIMPs, at the LHC and elsewhere, but no evidence for WIMPs has emerged. This motivates looking beyond the WIMP paradigm. There is no compelling reason for the dark sector to be simpler than the SM, particularly given there are five times more DM than SM particles. In general, dark sectors can feature new particles and forces with signatures not found in the WIMP scenario. One such new particle is a "dark photon".

In models with rich dark sectors, mediators coupled to SM via a "portal". The vector mediator is a massive dark photon $A^{\prime}$ that couples to the SM via "kinetic mixing" $\varepsilon$. A signature at colliders is $A^{\prime} \rightarrow \mu^{+} \mu^{-}$. The LHCb experiment explores the $\left[m\left(A^{\prime}\right), \varepsilon^{2}\right]$ dark photon parameter space with good sensitivity to low $m\left(\mu^{+} \mu^{-}\right)$that is expected for $A^{\prime}$. The search is split into two categories: "Prompt-like" covering $m\left(A^{\prime}\right)$ from the dimuon threshold to $70 \mathrm{GeV}$ and "Long-lived" where $214 \mathrm{MeV}<m\left(A^{\prime}\right)<350 \mathrm{MeV}$ and the $A^{\prime}$ decay vertex is displaced from the primary vertex.

Results from $\mathrm{LHCb}$ using $1.6 \mathrm{fb}^{-1}$ of $13 \mathrm{TeV}$ data from 2016 are shown in Figure 8 [17]. LHCb makes the first exclusion for the $10.6<m\left(A^{\prime}\right)<70 \mathrm{GeV}$ region and is comparable to best published results for $m\left(A^{\prime}\right)$ from $2 m_{\mu}$ to $0.5 \mathrm{GeV}$. For long-lived $A^{\prime}$, this represents the first exclusion not from a beam dump experiment. 


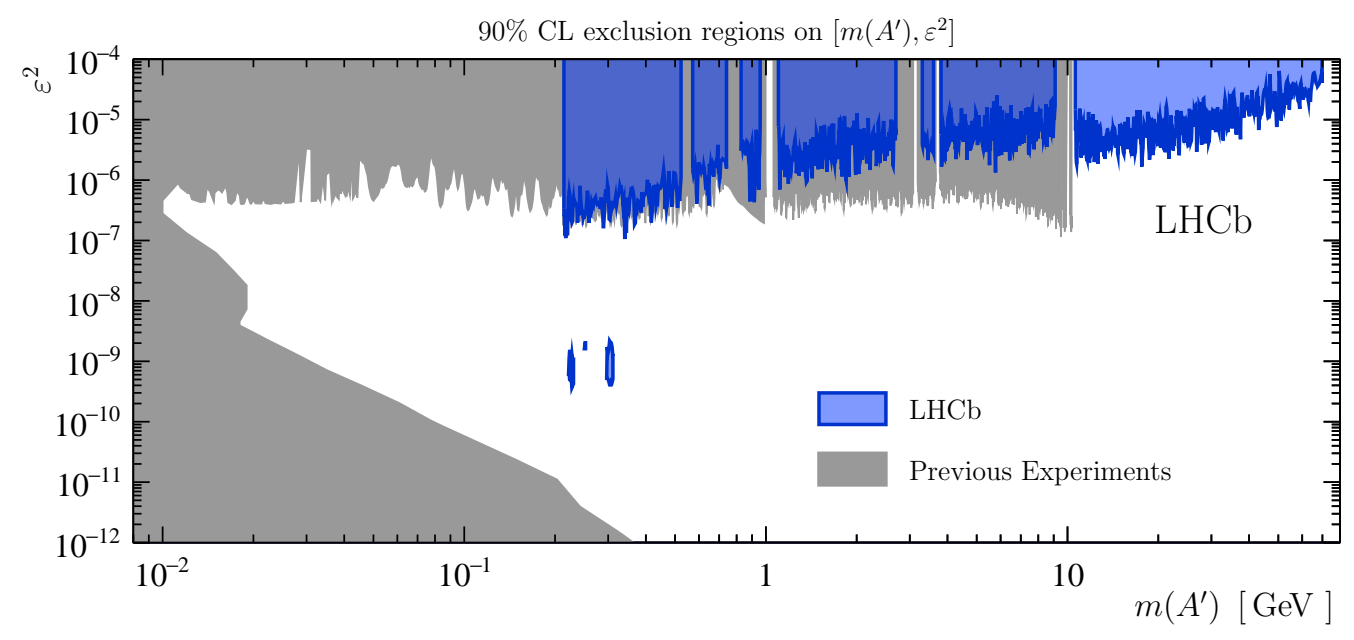

Figure 8: Exclusion in the mixing-dark photon mass plane from $\mathrm{LHCb}$ [17].

\section{Search in the Mono- $Z^{\prime}$ Channel}

The final search comes from ATLAS [18] and is a twist on the usual Mono- $X$ analyses. Here $X$ will represent a beyond-SM particle, namely a $Z^{\prime}$ that decays hadronically to two jets which can either be resolved or merged. Two (DM $+Z^{\prime}$ ) signal models arestudied: (1) a "dark-fermion" model where the $Z^{\prime}$ couples to a heavier dark-sector fermion $\chi_{2}$ and a lighter fermion $\chi_{1}$ which is the DM candidate and (2) a "dark-Higgs" model where the dark-sector Higgs boson $h_{D}$ is radiated from the $Z^{\prime}$ and $h_{D}$ decays to $\chi \bar{\chi}$. Examples of limits on the coupling of the $Z^{\prime}$ to quarks $g_{\mathrm{SM}}$ for these models are shown in Figure 9. The results are comparable to limits from dijet searches when $g_{\mathrm{DM}}=1$ is assumed.
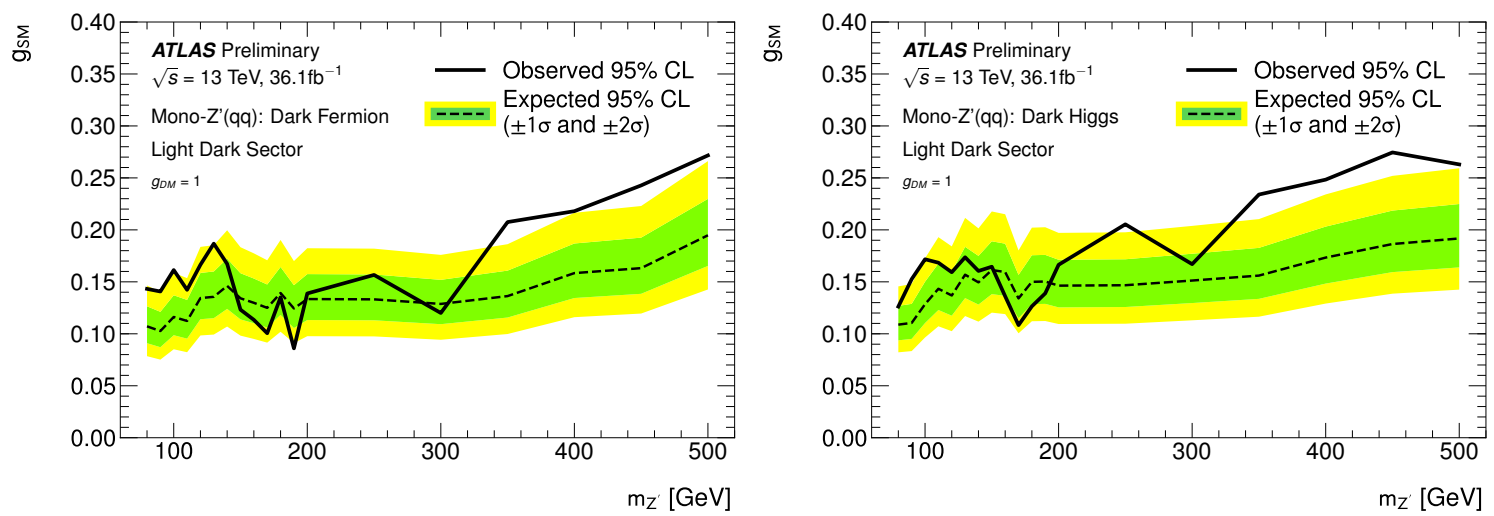

Figure 9: Limits from ATLAS [18] on the the mediator coupling to quarks $g_{\mathrm{SM}}$ (assuming $g_{\mathrm{DM}}=1$ ) in mono- $Z^{\prime}$ models vs. $m_{Z^{\prime}}$ for (left) a dark-fermion model and (right) a dark-Higgs Model.

\section{Summary and Outlook}

The ATLAS, CMS and LHCb experiments are actively searching for DM. There is sensitivity to DM under many model assumptions for the interaction and the mediator. No evidence for DM 
has been observed so far but there is much more phase space to be explored. In considering the outlook for DM searches at the LHC, it is important to keep in mind that only a small fraction of the total LHC data set is in hand to date, innovative analysis techniques are being developed and new directions in terms of models and signatures are continuously being dreamed up. The bottom line is that the LHC experiments are just getting started with DM searches.

\section{References}

[1] Planck Collaboration, Planck 2015 results. XIII. Cosmological parameters, Astron. Astrophys. 594 (2016) A13, arXiv:1502.01589 [astro-ph.CO].

[2] ATLAS Collaboration, The ATLAS Experiment at the CERN Large Hadron Collider, JINST 3 (2008) S08003.

[3] CMS Collaboration, The CMS experiment at the CERN LHC, JINST 3 (2008) S08004.

[4] LHCb Collaboration, The LHCb Detector at the LHC, JINST 3 (2008) S08005.

[5] J. Abdallah et al., Simplified models for dark matter searches at the LHC, Physics of the Dark Universe 9-10 (2015) 8-23, http://www.sciencedirect.com/science/article/pii/s2212686415000163.

[6] A. Albert et al., Recommendations of the LHC Dark Matter Working Group: Comparing LHC searches for heavy mediators of dark matter production in visible and invisible decay channels, arXiv:1703.05703 [hep-ex].

[7] CMS Collaboration, A. M. Sirunyan et al., Search for new physics in final states with an energetic jet or a hadronically decaying $W$ or $Z$ boson and transverse momentum imbalance at $\sqrt{s}=13 \mathrm{TeV}$, Phys. Rev. D97 (2018) 092005, arXiv:1712.02345 [hep-ex].

[8] ATLAS Collaboration, Search for dark matter and other new phenomena in events with an energetic jet and large missing transverse momentum using the ATLAS detector, JHEP 01 (2018) 126, arXiv:1711.03301 [hep-ex].

[9] CMS Collaboration, Search for invisible decays of the Higgs boson produced through vector boson fusion at $\sqrt{s}=13 \mathrm{TeV}$, CMS-PAS-HIG-17-023, Geneva, 2018, https://cds.cern. ch/record/2308434.

[10] ATLAS Collaboration, Summary plots from the ATLAS Exotic physics group, https: / / at las . web. cern. ch/At las / GROUP S / P HYS ICS / CombinedSummaryPlots / EXOTICS/.

[11] CMS Collaboration, Dark Matter Summary Plots from CMS for LHCP and EPS 2017, https://twiki.cern.ch/twiki/pub/CMSPublic/PhysicsResultsEXo/ DM-summary-plots-Jul17.pdf.

[12] CMS Collaboration, Search for low mass vector resonances decaying into quark-antiquark pairs in proton-proton collisions at $\sqrt{s}=13 \mathrm{TeV}$, JHEP 01 (2018) 097, arXiv:1710.00159 [hep-ex] .

[13] ATLAS Collaboration, Search for low-mass dijet resonances using trigger-level jets with the ATLAS detector in pp collisions at $\sqrt{s}=13 \mathrm{TeV}$, arXiv:1804.03496 [hep-ex] .

[14] CMS Collaboration, Search for dark matter produced in association with a top quark pair at $\sqrt{s}=13$ TeV, Tech. Rep. CMS-PAS-EXO-16-049, CERN, Geneva, 2018.

https://cds.cern.ch/record/2311235. 
[15] CMS Collaboration, Search for dark matter produced in association with a Higgs boson decaying to $\gamma \gamma$ or $\tau^{+} \tau^{-}$at $\sqrt{s}=13 \mathrm{TeV}$ with the CMS detector, Tech. Rep. CMS-PAS-EXO-16-055, CERN, Geneva, 2018. https: //cds. cern. ch/record/2308271.

[16] ATLAS Collaboration, ATLAS Collaboration, Search for Dark Matter Produced in Association with a Higgs Boson Decaying to b $\bar{b}$ using $36 \mathrm{fb}^{-1}$ of pp collisions at $\sqrt{\mathrm{s}}=13 \mathrm{TeV}$ with the ATLAS Detector, Phys. Rev. Lett. 119 (2017) 181804, arXiv:1707.01302 [hep-ex].

[17] LHCb Collaboration, Search for Dark Photons Produced in 13 TeV pp Collisions, Phys. Rev. Lett. 120 (2018) 061801, arXiv:1710.02867 [hep-ex].

[18] ATLAS Collaboration, Search for dark matter in events with a hadronically decaying vector boson and missing transverse momentum in pp collisions at $\sqrt{s}=13 \mathrm{TeV}$ with the ATLAS detector,

ATLAS-CONF-2018-005, 2018, http: / / cds. cern. ch/record/2311941. 